\section{Evaluation of in vivo denture plaque assessment methods}

\author{
L. Coulthwaite ${ }^{1}$ and J. Verran ${ }^{2}$
}

IN BRIEF
Provides a critical review of methods
used to assess coverage of denture
surfaces by plaque.
- Notes that there is no current
standardised method.
Considers the relative merits of
planimetric and visual indices.
Recommends the maintenance of an
image library as a record of data, and
blinded visual scoring from images as
current best practice.

\begin{abstract}
Background Measurement and assessment of denture plaque can provide valuable information regarding an individual's oral health status and assessment of new treatments or products. Current methods tend to rely on subjective indices or image analysis derived planimetric (area measurement) assessment of stained plaque on dentures. Plaque indices are most commonly used to assess plaque coverage without image capture. This is not ideal because the methods are subjective, examiner bias may occur, there is no reproducibility between studies, the methods have lower accuracy and sensitivity than image analysis, and there is no record. To the authors' knowledge, no standardised published method of denture plaque assessment is currently employed for product development and testing. Method In this study visual and planimetric plaque assessment methods were compared using reference dentures. In addition, an in vivo study compared these methods for evaluating denture cleanser efficacy. Results and conclusions The results show that blinded image scoring is more representative of the true plaque area coverage than 'live' denture scoring, detecting significant decreases in plaque coverage. Planimetric analysis provides a more sensitive and less subjective technique with greater differentiation between treatments. However, analysis is very time consuming. Thus, a number of recommendations are made regarding quantification of denture plaque for the assessment of cleanser products.
\end{abstract}

\section{INTRODUCTION}

Measurement and assessment of denture plaque can provide valuable information regarding oral health status associated with denture wearing and assessment of new treatments or products. The potential pathogenic aspects of denture plaque have been recently reviewed. ${ }^{1}$ Methods for assessing plaque quantity have included dry or wet weight measurement, biochemical assays, oxygen consumption assays, microbiological counts and visual indices or planimetric assessments (area measurement) of plaque coverage or biofilm thickness in situ..$^{2-7}$ Many scoring systems used for dentures have derived from studies on natural teeth, in which individual tooth plaque measurements have been used in

${ }^{1}$ Lecturer, ${ }^{2 *}$ Professor of Microbiology,

School of Biology, Chemistry and Health Science, Manchester Metropolitan University, Chester Street, Manchester, M1 5GD

${ }^{*}$ Correspondence to: Professor Joanna Verran

Tel: +44 (0)161 247 1206; Email: j.verran@mmu.ac.uk

Online article number E12

Refereed Paper - accepted 1 May 2009

DOI: $10.1038 /$ sj.bdj.2009.854

${ }^{\circledR}$ British Dental Journal 2009; 207: E12 epidemiological studies of periodontal disease. ${ }^{8-10}$ For clinical trials with denture cleansing products however, a broader approach can be taken, in that scores per denture surface are sufficient and appropriate to judge the efficacy of products. Current methods tend to rely on subjective or image analysis derived estimates of area coverage of dentures by stained plaque., ,, $11-13^{-13}$

For denture plaque assessment most methods assess plaque coverage by staining with a disclosing agent, most commonly erythrosine. Budtz-Jörgensen and Bertram ${ }^{11}$ proposed a simple index to grade plaque coverage on the denture fitting surface: excellent - none or only a few spots of plaque; fair $-<50 \%$ denture base covered; and poor $->50 \%$ denture base covered. A slightly more sensitive method was described in $1978^{14}$ where the amount of denture plaque on the fitting surface was graded $0=$ non-visible, $1^{+}=$less than one third covered, $2^{+}=$one third to two thirds covered, and $3^{+}=$more than two thirds covered. Augsberger and Elahi ${ }^{12}$ developed a well-used index for scoring denture plaque where the maxillary surface is sectioned into eight areas, four on the buccal surfaces and four on the fitting surface. The mean plaque score allocated is calculated from the sum of all eight sites: $0=$ no plaque, $1=$ light plaque (1-25\% area covered), $2=$ moderate plaque (26-50\% area covered), 3 = heavy plaque (51-75\% area covered), and 4 = very heavy plaque (76-100\% area covered). McCabe et $a l .{ }^{15}$ evaluated denture plaque coverage by reference to a series of standard dentures painted to simulate plaque scores in the 0-10 range and showed no significant difference in scores recorded by three operators. In an evaluation of this latter method, Shaloub and Addy ${ }^{6}$ suggested that area-based plaque indices can be scored accurately and with minimal variability by using laboratory models to train and assess examiners. In a recent study by Paranhos et al. ${ }^{16}$ the denture base fitting surface was divided into 14 areas and biofilm was quantified in each area on a score of $0=$ no visible biofilm, $1=$ isolated spots, 2 = coverage of less than half of the area, $3=$ coverage of more than half of the 
area, and $4=$ area completely covered with visible biofilm. Mean scores per denture were calculated. This latter method appears to be a more time consuming version of the Augsberger and Elahi index with no apparent advantages.

Mandibular prostheses have been reported to have a higher mean biofilm percentage area coverage compared to maxillary prostheses ${ }^{17}$ but the area is smaller. The maxillary dentures tend to receive more attention because they have a greater surface area for plaque accumulation between the denture and palatal mucosa and are more associated with denture stomatitis and the presence of Candida. Salles et al. ${ }^{17}$ measured the biofilm coverage by calculating the relationship between the biofilm area and total area of the internal base of the prostheses.

Most clinical plaque indices measure a variable on an ordinal scale (ranked in an order and counted, ie $1,2,3$, rather than measured), whereas an interval scale (measured as a difference between two units) is more powerful, providing more precise results and reducing clinical trial sizes $^{4}$ in addition to reducing subjectivity, increasing statistical strength and allowing better discrimination between similar products. Most clinical plaque indices yield ordinal data quickly, but are dependent upon a trained examiner's subjective evaluation ${ }^{18}$ and a clinician to conduct examinations, increasing clinical trial costs. Plaque indices are most commonly used to assess denture plaque coverage without image capture. This is not ideal because the methods are subjective, examiner bias may occur, there is no reproducibility between studies and the methods have lower accuracy and sensitivity than image analysis. Overall, because of the variety of scoring methods and lack of standardisation between laboratories, it is often difficult to make comparisons between the efficacies of denture cleansing products. ${ }^{7,15}$

Planimetric plaque systems are increasingly being used to measure plaque surface area, usually expressed as a percentage plaque index (PPI), representing the percentage of the tooth covered by disclosed plaque. The basic principle involves storing an image of the tooth and using computer software to measure the surface area in pixels. This method has progressively become more computerised and
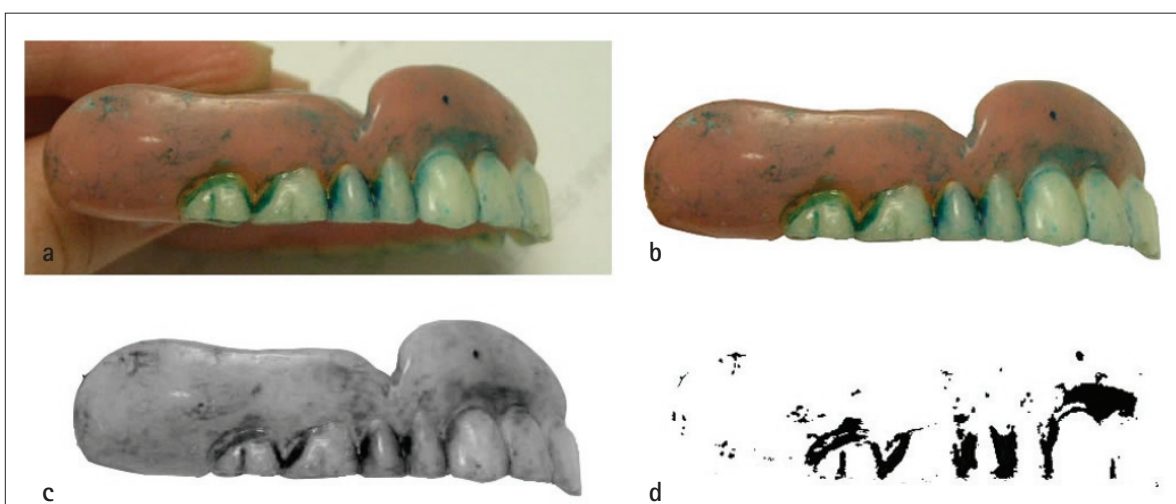

C

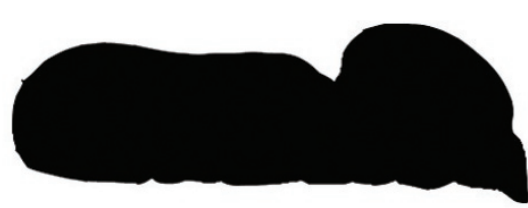

e

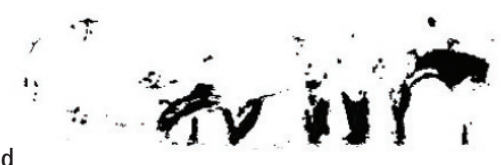

Fig. 1 Planimetric assessment of denture plaque method 1. a) Disclosed image as JPG file; b) denture area selected, copied and saved; c) red colour channel selected (threshold level 30), plaque area selected then 'select similar' to highlight all the plaque; d) plaque area filled with black, copied and saved; e) total denture area filled with black and saved. Files d) and e) measured in Scion image as total number of black pixels. Percentage plaque pixels from total denture surface pixels gives percentage area cover of plaque on the denture surface

less subjective. Digital photography most commonly uses the RGB (red, green, blue) colour space for colour encoding, which specifies the proportions and intensities that produce a particular colour. Successful planimetric analysis requires high quality images with fine focus, absence of flash artefacts, standardised size of images and clear separation of image components that can be distinguished by the software. ${ }^{19}$ Absorption, colour and intensity of disclosing dyes may be influenced by interactions with residues of denture cleansing agents, ${ }^{20}$ thus the cleanser activity mechanism should be considered before selecting an appropriate disclosing agent. Digitised images can be stored and examined at a later time, allowing longitudinal comparisons, re-measuring for reproducibility and double-blind studies. ${ }^{4,21}$

Numerous studies have found computerbased plaque assessment more reliable $\mathrm{e}^{2,4,6,22}$ and more objective than classic plaque indices. $^{22}$ This technique offers linear measurements, increased reproducibility, ${ }^{4}$ increased objectivity ${ }^{2}$ and increased discriminating power. ${ }^{23}$ The technique also has good correlation with plaque weight, viable microbial counts and visual assessment. ${ }^{2}$ Development of automated systems for image capture and plaque scoring reduce investigator involvement and subjectivity. ${ }^{21}$ To the authors' knowledge, no standardised method of denture plaque assessment is currently employed for product development and testing studies, or at least has not been published.

\section{AIMS}

The aim of this study was to compare current available visual and planimetric plaque measurement techniques for ease of measurement, accuracy, reproducibility and relationships, in order to recommend the 'best' method for standardisation and future general dental practice.

\section{MATERIALS AND METHODS}

\section{Visual assessment}

Denture plaque may be assessed using a visual index, allocating plaque cover scores with reference to a series of ten standard denture sets. In this study, the dentures had been prepared by painting with an acrylic varnish to which had been added a quantity of blue dye designed to mimic scores on the 0-10 scale of plaque as described by McCabe et al..$^{15}$ Three images of each standard maxillary denture (fitting, buccal right, buccal left) were captured using standard white-light flash photography (Minolta DIMAGE F100). The three surfaces of each denture - fitting, teeth and polished surfaces - were scored by a single examiner, and each denture was given a 
score (0-10) reflecting the average of the three surfaces. This visual scoring system was compared to three planimetric plaque analysis methods.

\section{Planimetric assessment}

The images were processed in Adobe Photoshop (version 7; Adobe Systems Inc.). The denture area was selected using the 'magnetic lasso' tool, copied, pasted and saved (Figs 1a and 1b). In the red colour channel (Fig. 1c), the image plaque area was highlighted using the 'magic wand' tool (tolerance level 8), then the 'select similar' tool (threshold level 30) until all plaque was selected. The plaque area was filled in black, copied and saved (Fig. 1d). The total denture area was filled with black and saved (Fig. 1e). The images were opened in an image analysis package (Scion Image vB4.03, Scion Corp, USA) and the total number of black pixels measured for each image. The percentage plaque index (PPI) from the area coverage on the denture can be calculated from the pixel counts of these images (total plaque pixels/total denture surface pixels).

Plaque area coverage was measured using three different planimetric analysis methods. In method one the total plaque area coverage was measured as described above. In method two the total plaque area coverage was measured from each image divided into surfaces as described above for the visual scoring, ie fitting, teeth, polished. The fitting surface image was analysed as in method one. The right and left buccal surface images were each separated into two further images to highlight polished surfaces and teeth independently and a mean PPI calculated. In method three, the total plaque was measured from images divided according to the index method of Augsberger and Elahi. ${ }^{12}$ Eight sites on the denture, four on the buccal surfaces and four on the palatal or fitting surface were assessed by planimetric analysis and visual scoring. The tissue-fitting surface of each maxillary denture was divided into four sections approximately equal in area. The quantity of plaque present in each area was visually scored according to the method of Augsberger and Elahi ${ }^{12}$ as follows: 0 = no plaque; 1 = light plaque (1-25\% area covered); $2=$ moderate plaque (26-50\% area covered); 3 = heavy plaque (51-75\% area covered); and $4=$ very heavy plaque (76-100\% area covered). The mean plaque score from all eight sites was calculated. All denture images were analysed by a single examiner. A single planimetric method was selected based on the results obtained and this was used for subsequent analyses in the clinical study.

\section{Clinical plaque assessment study}

An in vivo clinical study assessed the use of white light images for analysis of plaque area coverage. The study was conducted in accordance with the declaration of Helsinki (1964) and subsequent amendments. Ethical approval was obtained before the start of the study from the appropriate in-house Ethical Committee at the commercial study centre, and additional ethical approval was granted by Manchester Metropolitan University for all image and data analyses. All volunteers were provided with information sheets and signed, witnessed informed consent was obtained. Volunteers were advised of their right to withdraw from the study at any time.

Eight volunteers were recruited from an established denture panel (via a recruitment agency) with complete maxillary and mandibular polymethylmethacrylate (PMMA) dentures. The dentures were rinsed to remove any loose food particles and stained with methylene blue disclosing solution (FDC Blue \#1, 0.25\% in deionised water) for $1 \mathrm{~min}$ and then rinsed before visual scoring (as described earlier) and image capture using a digital camera
(Minolta DIMAGE F100) and white light illumination. Three images of each denture were captured (fitting, buccal right, buccal left). The maxillary and mandibular dentures were allocated a treatment or control procedure based on arrival order. Both dentures were brushed gently for $10 \mathrm{~s}$ on each surface under running water (control) and then one denture (treatment) was additionally immersed for 10 min in Steradent effervescent tablets (Reckitt Benckiser, Hull, UK). Following treatment/control procedures, dentures were rinsed in running water and restained with methylene blue for repeat scoring and imaging. A single examiner carried out the denture treatment/control procedure, another examiner visually scored the dentures and an independent examiner obtained denture images. Image orders were randomly assigned (blinded) and visually scored at a later date by the same examiner used for live visual scoring. Visual scores and planimetric plaque measurements were compared. The threshold function in Photoshop was selected, and the area of plaque was measured at three different, pre-determined thresholds. These related to the darkness of the plaque visible on the image and thus may offer an opportunity to include a depth component to the technique.

\section{Statistical analyses}

The denture plaque scores were analysed using the one-way analysis of variance

\section{Table 1 Denture standard scores, mean of the three planimetric analysis methods and} reordered standard scores based on quantification of the mean plaque area coverage

\begin{tabular}{l|l|l|l|l}
\hline $\begin{array}{l}\text { Prepared denture } \\
\text { standard }\end{array}$ & Mean \% area coverage & \pm SD & $\begin{array}{l}\text { Reordered denture } \\
\text { standard }\end{array}$ & $\begin{array}{l}\text { Actual } \\
\text { score* }\end{array}$ \\
\hline 1 & 0.62 & 0.13 & 1 & 1 \\
\hline 2 & 6.26 & 1.28 & 2 & 1 \\
\hline 3 & 9.23 & 0.26 & 3 & 1 \\
\hline 4 & 20.55 & 1.01 & 7 & 3 \\
\hline 5 & 17.33 & 3.89 & 6 & 2 \\
\hline 6 & 12.67 & 1.93 & 4 & 2 \\
\hline 7 & 25.89 & 2.56 & 8 & 3 \\
\hline 8 & 14.64 & 2.19 & 5 & 2 \\
\hline 9 & 36.67 & 3.65 & 9 & 4 \\
\hline 10 & 70.45 & 5.31 & 10 & 8 \\
\hline
\end{tabular}


and application of Tukey's HSD multiple comparisons test to reveal any statistical significance between scores. The three planimetric methods of percentage coverage were compared to the $0-10$ visual scores and assessed for agreement and significant differences using ANOVA in Minitab (v.14) statistical software.

\section{RESULTS}

It is apparent that the stained denture standards did not represent an accurate scale or wide range of plaque area coverage (Table 1). Percentage plaque area coverage on the ten denture standard sets was calculated using three different image analysis methods. The methods showed good agreement, no significant differences and low standard deviations (Table 1, Fig. 2). There was greater coverage of plaque on the fitting surface than on the teeth or polished surfaces. The allocated denture scores (0-10) are not representative of the actual plaque area (Table 1). Using a $0-100 \%$ cover obtained via image analysis and converting to a 1-10 scale, real scores were assigned ( $1=1-10 \%, 2=11-20 \%$, scaled in series until index $10=91-100 \%$ ) showing a narrow distribution of plaque coverage (Table 1), which does not give sufficient differences between the scores. The Augsberger and Elahi index ${ }^{12}$ was more representative of actual plaque area and was less subjective than using the denture sets.

Planimetric analysis is very time consuming. If employed in a clinical study, method 1 requires analysis of 12 images (three per denture, pre- and post-treatment), method 2 requires analysis of 20 images (five per denture, pre- and posttreatment) and method 3 requires analysis of 32 images (eight per denture, pre- and post-treatment). Method 1 took on average $2 \mathrm{~h}$ for analysis of 12 images, with the other two methods taking longer. There was no significant difference between the mean PPI scores from the methods, thus method 1 was selected for the clinical studies because of the shorter analysis time. In all cases, the images provide a resource for storage. The analysis could be carried out at a subsequent time and the images provided a reference library for quality assurance purposes.

In the clinical study, white-light images of dentures were captured before and after

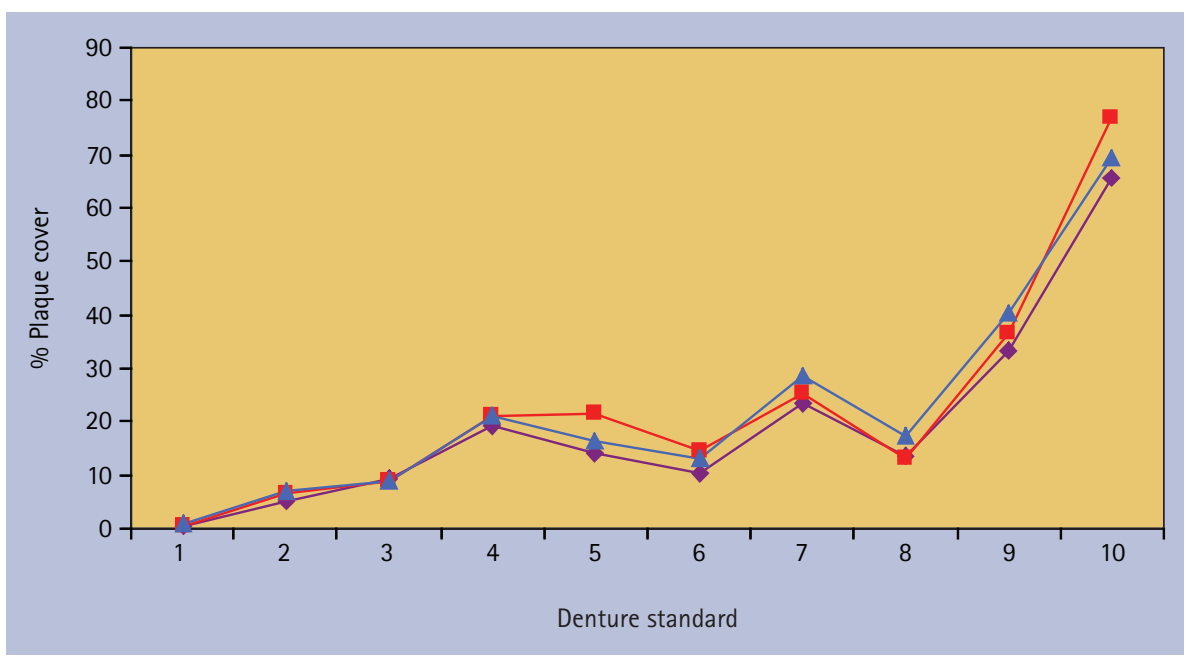

Fig. 2 Percentage plaque area cover on the denture standards from planimetric analysis methods $1(\diamond), 2(\square)$ and $3(\Delta)$ showing little difference between the three methods
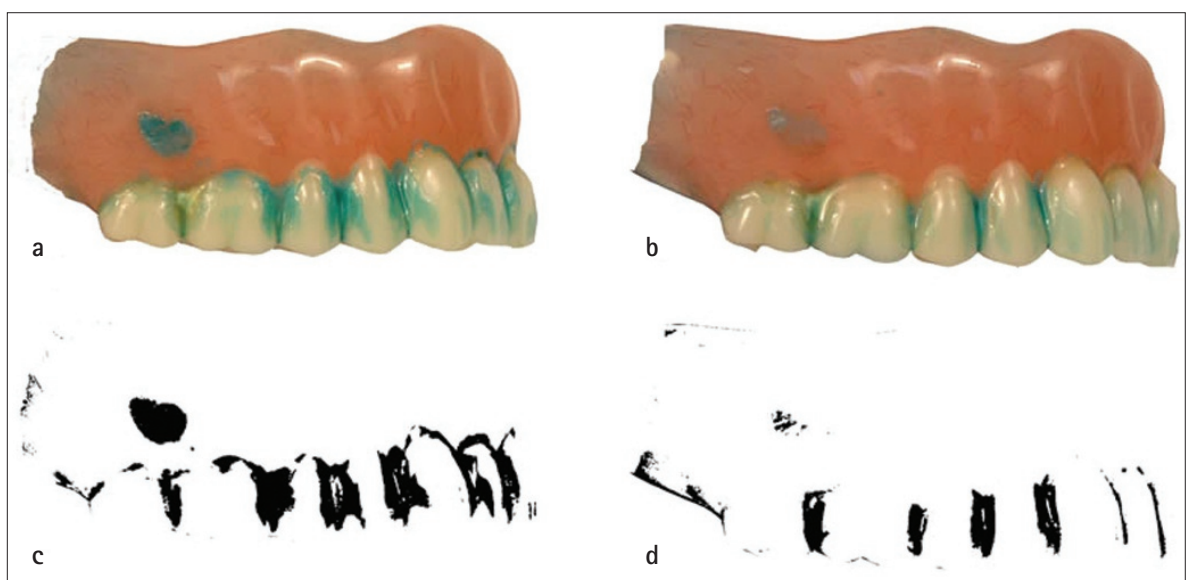

Fig. 3 Images of a maxillary denture disclosed with methylene blue before $(a, c)$ and after $(b, d)$ treatment with a denture cleanser

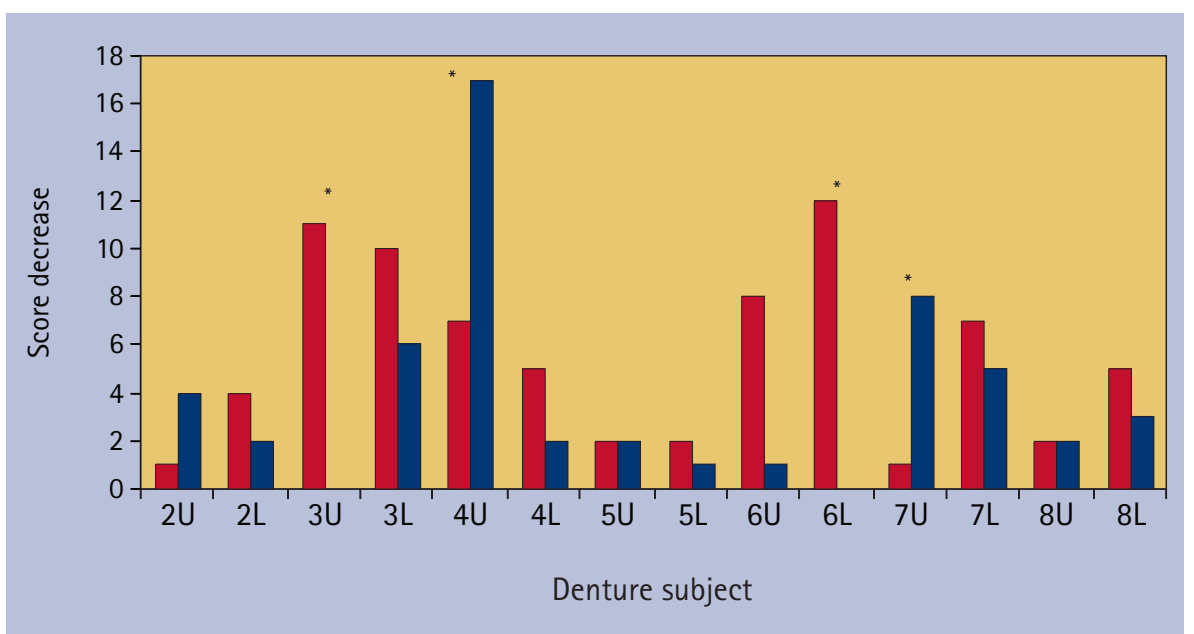

Fig. 4 Decreases in plaque coverage scores (ie removal efficacy) from dentures stained before and after control brushing or treatment with an effervescent tablet. Maximum score of plaque on three surfaces is 30 . Plaque scores from stained dentures during the trial ( $\square$ ) and from images taken ( $\square$ ) are compared. Maxillary denture (U) and mandibular denture (L). *Significant differences in plaque decreases between live and blinded image scores

treatment/control procedures (Fig. 3). A single examiner made a visual assessment of plaque before and after treatment and the same examiner scored randomised images of the dentures at a later date for comparison of assigned scores, expressed as plaque score decreases in Figure 4. Both control and treatment procedures reduced 


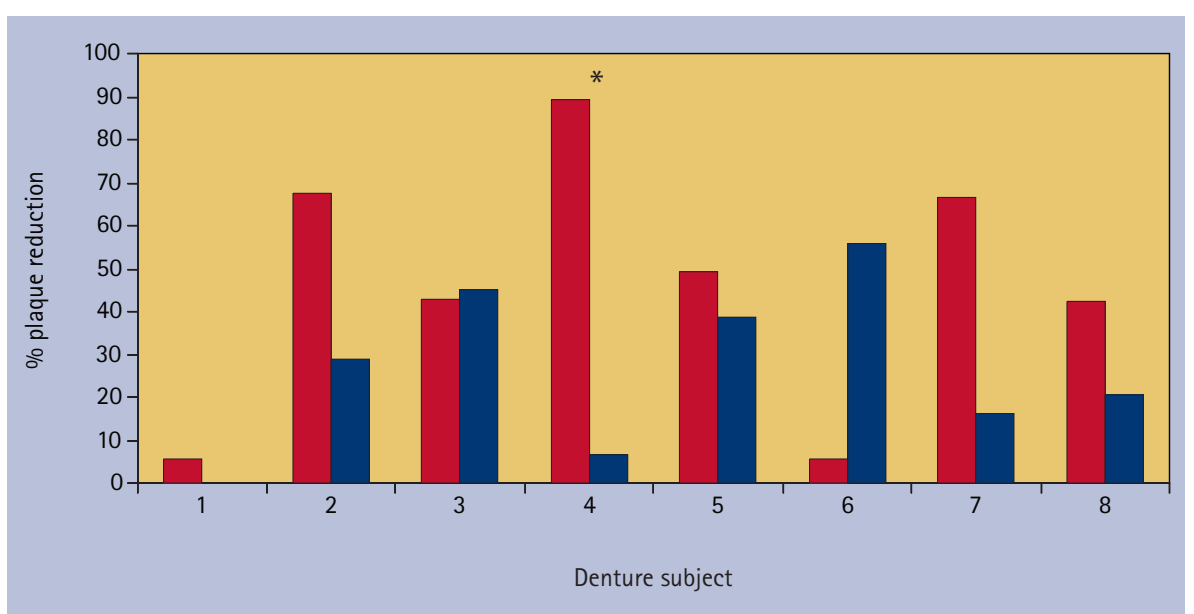

Fig. 5 Percentage plaque area reduction on maxillary ( $\square$ ) and mandibular ( $\square$ ) dentures from eight subjects using total plaque coverage scores from pixel counts of disclosed plaque on the buccal and fitting surfaces of dentures. *Significant differences in plaque reduction on the treated compared to control denture

plaque area coverage on the dentures. In six of the eight subjects, plaque reduction was greater on the maxillary compared to mandibular denture, which implied a greater potential for plaque decrease due to the larger surface area of the maxillary denture and had more influence on plaque reduction than the treatment/ control procedure. The live visual scores showed a significant decrease in score on the treated compared to control denture of subject $3(t=4.24, p=0.013)$, although overall Steradent showed greater plaque reduction on half of the dentures compared to the control.

In a larger 'live' plaque scoring study (data not shown), Steradent produced greater plaque reductions than the control in 31 of the 38 subjects. The blinded image visual scores showed a significant decrease on the treated compared to control denture only for subject $4(t=6.71$, $\mathrm{p}=0.003)$, although Steradent showed greater plaque reduction on a further three out of the eight dentures compared to the control. The visual scores do not agree for the live (not blind) and blind analyses and significant differences were obtained between these two analyses for four of the eight subjects $(3,4,6,7 ; p<0.05)$. There was no significant difference in maxillary and mandibular plaque scores from the live assessment. There was a significant difference from the blind image scores, where greater plaque coverage was recorded from the fitting surface ( $\mathrm{F}=8.94, \mathrm{p}<0.001)$.

Plaque was measured by planimetric analysis method 1 and expressed as percentage plaque area reduction (Fig. 5).
Plaque area was significantly decreased after treatment with Steradent compared to the control in one subject (subject 4), where an $89 \%$ reduction in plaque coverage was obtained. This supports the blind image visual scores. Although overall, Steradent reduced plaque coverage by greater than $40 \%$ in half of the subjects, results show that accurate plaque area measurement compared to visual scoring did not reveal greater significant differences in plaque coverage between control and treated dentures. However, both the control and treated dentures were cleaned by brushing, hence plaque reduction was expected on both dentures. No assessment of cultivability was made in this study, where a significantly greater 'kill' would be expected from Steradent.

The tolerance threshold level was set at 30 for selecting plaque areas in the red colour channel; however tolerance setting is subjective depending on the initial plaque colour intensity (which may indicate a potential for indirect measurement of plaque depth by colour intensity). The software had difficulty detecting disclosed plaque on the denture fitting surface due to the paler colour of the acrylic on this surface and the irregular surface features creating shadows on the image.

\section{DISCUSSION}

\section{Evaluation of reference denture standards for plaque coverage}

The scores allocated in the denture standard set do not correlate well with the percentage plaque area coverage calculated by three image analysis methods. This demonstrated that visual scoring is, by definition, subjective. Visual scoring methods require examiner training. The planimetric techniques require considerably more time for analysis but require little examiner training other than in patience, knowledge of image analysis software packages and a steady hand for drawing outlines. Planimetric analysis offers improved accuracy, decreased subjectivity and archivable images for further analysis or longitudinal studies. There was no significant difference between the three planimetric methods in terms of plaque area measurement, however assessment of entire surfaces (method one) required the least image processing and analysis time, thus was the method of choice for further assessments.

\section{Assessment of denture cleanser efficacy in a clinical study}

Planimetric analysis of plaque coverage revealed that the blinded image scoring was more representative of the true plaque area coverage than 'live' denture scoring. Blinded image scoring detected significant decreases in plaque coverage, which were confirmed by planimetric assessment. Planimetric analysis provides a more sensitive and less subjective technique with greater differentiation between similar cleansing products. However, analysis is very time consuming and still subjective to an extent, relying on examiner interpretation in threshold setting and defining borders.

Planimetric assessment revealed a reduction in plaque coverage for both treatments. However, dentures were initially brushed, hence plaque coverage reduction would be expected: there was some evidence of an improved effect using Steradent. Difficulties in the study included the patients acting as their own controls since the plaque coverage on maxillary and mandibular dentures can differ considerably, thus the potential for plaque reduction was affected by the study design. Also, the dentures have different surface areas, the maxillary being larger and generally having more irregular features for plaque retention, making physical plaque removal difficult. The method used supports the British Dental Association recommendations to brush and soak dentures. 'Live' plaque scoring in the study was subjective, since the examiner 
was present in the room where dentures were being processed and the treated denture had a residual 'clean' smell from the cleanser solution. Treatment with Steradent in comparison to the control significantly decreased the viable counts of facultative anaerobes in denture fitting surface plaque from 67\% of subjects (unpublished data). The assessment of denture cleanser efficacy for plaque removal should thus be supported by antimicrobial activity assessment for testing new products and formulations.

Differences were observed in coverage between maxillary and mandibular dentures (mandibular having a greater percentage plaque coverage overall), but were not significant. Plaque scores on denture fitting surfaces were significantly higher than on polished surfaces from the blind image scores, in agreement with previous studies. ${ }^{15,24}$ Greater plaque coverage on the fitting surface was expected since this surface is more difficult to clean due to the uneven surface features allowing plaque retention. Indeed, the control of fitting surface plaque is important for clinical reasons, while for tooth surfaces aesthetics have a role to play.

Planimetric methods are less subjective and provide greater statistical significance over visual indices. Image analysis software is currently is being developed, which will reduce the subjectivity and time and labour intensive input currently required for planimetric analysis by using automated edge detection ${ }^{25}$ to differentiate plaque from the denture. ${ }^{26}$

\section{CONCLUSIONS}

The process of planimetric plaque analysis is complicated and time consuming, reinforcing the importance of traditional visual scoring methods as the gold standards in this area of dentistry. Based on the results obtained in these studies a number of recommendations with regard to assessment and quantification of plaque on dentures for the assessment of cleanser product treatments may been made:
- Discard the denture standard models used in this study

- Create new standards based on known coverage information and creating images or models of known specific percentage plaque coverage on dentures for reference, using a wider range and distribution of coverage than that used in this study

- Take white light images of disclosed dentures for archiving using either a good quality digital camera or preferably an image capture system with a CCD video camera for capture of images as seen live on screen. Use a camera copy stand and constant white light illumination

- Blinded visual scoring of images should be included as the gold standard using the method of Augsberger and Elahi ${ }^{12}$ (which gave the lowest variance from the comparison of planimetric analysis methods with the denture standards). If desired, planimetric analysis can be done on the same images at a later date, adding no extra time to the clinical study.

It is envisaged that with these developments the gold standard would be moved from a subjective grading system to a sophisticated accurate, reliable, reproducible and objective partially automated plaque quantification system.

The authors wish to thank Manchester Metropolitan University and Reckitt Benckiser Healthcare UK for jointly sponsoring this work.

1. Coulthwaite L, Verran J. Potential pathogenic aspects of denture plaque. Br J Biomed Sci 2007; 64: 180-189.

2. Verran J. Rocliffe M D. Feasibility of using automatic image analysis for measuring dental plaque in situ. J Dent 1986; 14: 11-13.

3. Cardash H, Rosenberg M. An innovative method of monitoring denture hygiene. J Prosthet Dent 1990; 63: 661-664.

4. Soder P-O, Jin L J, Soder B. Computerized planimetric method for clinical plaque measurement. Scand J Dent Res 1993; 101: 21-25.

5. McCabe J, Murray D I, Kelly P J. The efficacy of denture cleansers. Eur J Prosthodont Restor Dent 1995; 3: 203-207.

6. Shaloub A, Addy M. Evaluation of accuracy and variability of scoring-area-based plaque indices.
J Clin Periodontol 2000; 27: 16-21.

7. Sheen $S_{1}$ Harrison A. Assessment of plaque prevention on dentures using an experimental cleanser. J Prosthet Dent 2000; 84: 594-601.

8. Quigley G A, Hein J W. Comparative cleansing efficiency of manual and power brushing. J Am Dent Assoc 1962; 65: 26-29.

9. Turesky S, Gilmore N D, Glickman I. Reduced plaque formation by the chloromethyl analogue of victamine C. J Periodontol 1970; 41: 41-43.

10. Silness J, Loe H. Periodontal disease in pregnancy. II. Correlation between oral hygiene and periodontal condition. Acta Odontol Scand 1964; 22: 121-135.

11. Budtz-Jörgensen E, Bertram U. Denture stomatitis. I. The etiology in relation to trauma and infection. Acta Odontol Scand 1970; 28: 71-92.

12. Augsberger $R$, Elahi J M. Evaluation of seven proprietary denture cleansers. J Prosthet Dent 1982; 47: 356-359.

13. Nikawa H, Hamada T, Yamashiro H, Kumagai H. A review of in vitro and in vivo methods to evaluate the efficacy of denture cleansers. Int J Prosthodont 1999; 12: 153-159.

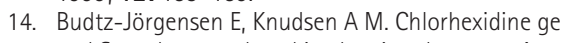
and Steradent employed in cleaning dentures. Acta Odontol Scand 1978; 36: 83-87.

15. McCabe J F, Murray D I, Laurie J, Kelly P J. A method for scoring denture plaque. Eur J Prosthodont Restor Dent 1996; 4: 59-64.

16. Paranhos H F O, Silva-Lovato C H, Souza R F, Cruz P C, Freitas K M, Peracini A. Effects of mechanical and chemical methods on denture biofilm accumulation. J Oral Rehabil 2007; 34: 606-612.

17. Salles A E S, Macedo LD, Fernandes R A G, SilvaLovato $\mathrm{CH}$, Paranhos H F O. Comparative analysis of biofilm levels in complete upper and lower dentures after brushing associated with specific denture paste and neutral soap. Gerodonto/ 2007; 24: 217-223.

18. Sagel P, Lapujade P G, Miller J M, Sunberg R J. Objective quantification of plaque using digital image analysis. Monogr Oral Sci 2000; 17: 130-143

19. Rekola M., Scheinin A. Quantification of dental plaque through planimetric analysis. Scand J Dent Res 1977; 85: 51-55.

20. Taaffe A, Riggott J M. A gravimetric technique for measurement of denture hygiene. J Dent 1985; 13: 271-276.

21. Carter K, Landini G, Walmsley A D. Automated quantification of dental plaque accumulation using digital imaging. J Dent 2004; 32: 623-628.

22. Smith $R$ N, Brook $A$ H, Elcock $C$. The quantification of dental plaque using an image analysis system: reliability and validation. J Clin Periodontol 2001: 28: $1158-1162$.

23. Quirynen M, Dekeyser C, Van Steenberghe D. Discriminating power of five plaque indices. J Periodontol 1991; 62: 100-105.

24. Budtz-Jörgensen $E$, Theilade $E$, Theilade J. Quantitative relationship between yeast and bacteria in denture induced stomatitis. Scand J Dent Res 1983; 91: 134-142.

25. Williams I, Bowring N, Guest E, Twigg P, Fan Y, Gadsby D. A combined statistical/neural network multi-scale edge detector. In Proceedings of the 5th IASTED International Conference on Visualisation, Imaging and Image Processing, September 7-9 2005, Benidorm, Spain. Calgary: Acta Press, 2005.

26. Coulthwaite L, Bowring N, Williams I, Verran J. Evaluation of novel methods for image analysis of denture plaque. J Dent Res 2008; 87(Spec Iss C): Abstract 0641 (PEF IADR). http://iadr.confex.com/ iadr/pef08/techprogram/abstract_111477.htm. 